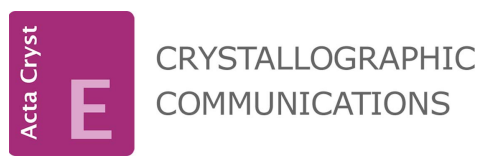

ISSN 2056-9890

Received 6 May 2015

Accepted 14 May 2015

Edited by J. Simpson, University of Otago, New Zealand

Keywords: crystal structure; synchrotron radiation; ethane-1,2-diamine; thiocyanate; transgeometry; chromium(III) complex; hydrogen bonds

CCDC reference: 1400767

Supporting information: this article has supporting information at journals.iucr.org/e

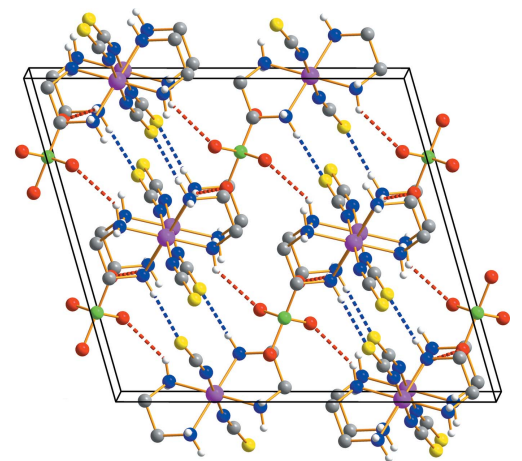

OPEN $\odot$ ACCESS

\section{Crystal structure of trans-bis(ethane-1,2-diamine- $\left.\kappa^{2} N, N^{\prime}\right)$ bis (thiocyanato- $\kappa N$ )chromium(III) perchlorate from synchrotron data}

\author{
Dohyun Moon ${ }^{\mathrm{a}}$ and Jong-Ha Choi ${ }^{\mathrm{b} *}$
}

${ }^{\mathbf{a}}$ Pohang Accelerator Laboratory, POSTECH, Pohang 790-784, Republic of Korea, and ${ }^{\mathbf{b}}$ Department of Chemistry, Andong National University, Andong 760-749, Republic of Korea. *Correspondence e-mail: jhchoi@anu.ac.kr

The structure of the title compound, $\left[\mathrm{Cr}(\mathrm{NCS})_{2}\left(\mathrm{C}_{2} \mathrm{H}_{8} \mathrm{~N}_{2}\right)_{2}\right] \mathrm{ClO}_{4}$, has been determined from synchroton data. The asymmetric unit consists of one half of a centrosymmetric $\mathrm{Cr}^{\mathrm{III}}$ complex cation and half of a perchlorate anion with the $\mathrm{Cl}$ atom on a twofold rotation axis. The $\mathrm{Cr}^{\mathrm{III}}$ ion is coordinated by the four $\mathrm{N}$ atoms of two ethane-1,2-diamine (en) ligands in the equatorial plane and two N-bound thiocyanate $\left(\mathrm{NCS}^{-}\right)$anions in a trans-axial arrangement, displaying a slightly distorted octahedral geometry with crystallographic inversion symmetry. The $\mathrm{Cr}-\mathrm{N}(\mathrm{en})$ bond lengths are in the range 2.053 (16)-2.09 (2) $\AA$, while the $\mathrm{Cr}-$ $\mathrm{N}$ (thiocyanate) bond length is 1.983 (2) $\AA$. The five-membered en rings are disordered over two sites, with occupancy ratios of 0.522 (16):0.478 (16). Each $\mathrm{ClO}_{4}{ }^{-}$anion is disordered over two sites with equal occupancy. The crystal structure is stabilized by intermolecular hydrogen bonds involving the en $\mathrm{NH}_{2}$ groups as donors and perchlorate $\mathrm{O}$ and thiocyanate $\mathrm{S}$ atoms as acceptors.

\section{Chemical context}

Considerable attention has been focussed for some time on metal complexes containing thiocyanate ligands because of their ability to coordinate through either the $\mathrm{N}$ or $\mathrm{S}$ atoms. Ethane-1,2-diamine (en) can coordinate to a central metal ion as a bidentate ligand via the two $\mathrm{N}$ atoms, forming a fivemembered chelate ring. The $\left[\mathrm{Cr}(\mathrm{NCS})_{2}(\mathrm{en})_{2}\right]^{+}$cation can form either trans or cis geometric isomers. Trans and cis isomers of the complex cation with $\mathrm{SCN}^{-}$or $\mathrm{ClO}_{4}{ }^{-}$counter-anions have been prepared and their IR spectral properties reported (House, 1973; Sandrini et al., 1978; De et al., 1987). IR and electronic spectral properties are useful in determining the geometric isomers of chromium(III) complexes with mixed ligands (Choi, 2000; Choi et al., 2004; Choi \& Moon, 2014). However, it should be noted that the geometric assignments based on spectroscopic studies are not always definitive.

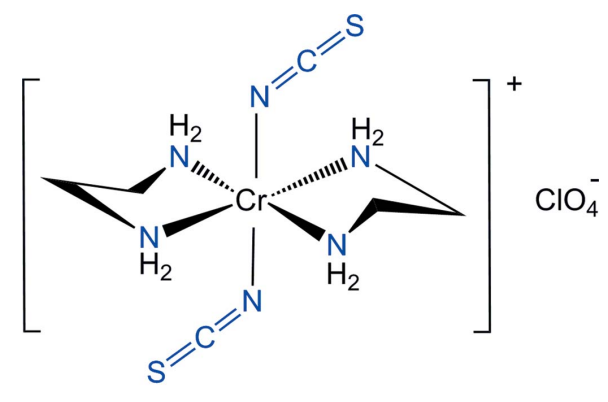

In a recent publication, we described the synthesis and crystal structure of trans-[Cr(NCS $\left.)_{2}(\mathrm{en})_{2}\right]_{2}\left[\mathrm{ZnCl}_{4}\right]$ (Moon \& 


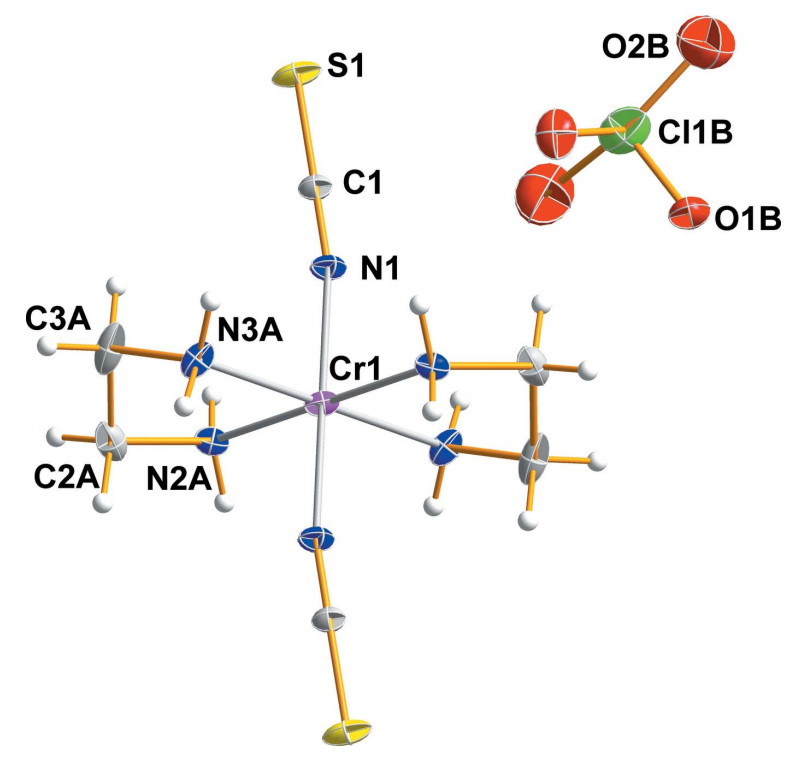

Figure 1

The molecular structure of (I), drawn with $20 \%$ probability displacement ellipsoids. Atoms of the minor disorder components have been omitted for clarity.

Choi, 2015). The asymmetric unit of this complex contained four halves of centrosymmetric $\left[\mathrm{Cr}(\mathrm{NCS})_{2}(\mathrm{en})_{2}\right]^{+}$complex cations and one $\left[\mathrm{ZnCl}_{4}\right]^{2-}$ anion. To compare and contrast this structure with a complex of this cation with a different counter-anion we report here the structure of trans-[Cr$(\mathrm{NCS})_{2}(\mathrm{en})_{2} \mathrm{ClO}_{4}$, (I).

\section{Structural commentary}

Fig. 1 shows an ellipsoid plot of trans-[Cr(NCS $\left.)_{2}(\mathrm{en})_{2}\right] \mathrm{ClO}_{4}$, (I), with the atom-numbering scheme. In the structure of (I), there is a centrosymmetric $\mathrm{Cr}^{\mathrm{III}}$ complex cation with two en ligands bound through their $\mathrm{N}$ atoms in equatorial sites and the two axial N-bound thiocyanate anions in a trans configuration. The asymmetric unit is composed of half of one complex cation and half a $\mathrm{ClO}_{4}{ }^{-}$anion. The $\mathrm{Cr}^{\mathrm{III}}$ atom is located on a crystallographic centre of symmetry, so this complex cation has molecular $C_{i}$ symmetry, while the the $\mathrm{Cl}$ atom of the perchlorate anion lies on a twofold rotation axis. The bidentate en ligand adopts a stable gauche conformation similar to that observed in related compounds (Brenčič \& Leban, 1981; Choi et al., 2010). The $\mathrm{Cr}-\mathrm{N}$ bond lengths for the en ligand range from 2.053 (16) to 2.09 (2) $\AA$, and these bond lengths are in good agreement with those observed in trans- $\left[\mathrm{CrF}_{2}(\mathrm{en})_{2} \mathrm{ClO}_{4} \quad(\right.$ Brenčič \& Leban, 1981), trans$\left[\mathrm{CrBr}_{2}(\text { en })_{2}\right] \mathrm{ClO}_{4}$ (Choi et al., 2010), trans- $\left[\mathrm{CrCl}_{2}\left(\mathrm{Me}_{2} \mathrm{tn}\right)_{2}\right]_{2^{-}}$ $\mathrm{ZnCl}_{4}\left(\mathrm{Me}_{2} \mathrm{tn}=2\right.$,2-dimethylpropane-1,3-diamine; Choi et al., 2011) and trans-[ $\left[\mathrm{CrF}_{2}(2,2,3\right.$-tet) $] \mathrm{ClO}_{4}$ (2,2,3-tet $=1,4,7,11$ tetraazaundecane; Choi \& Moon, 2014). The $\mathrm{Cr}-\mathrm{N}$ (thiocyanate) bond length is 1.983 (2) $\AA$ and is similar to the average values of $1.985(2), 1.995(6), 1.983(2)$ and 1.996 (15) $\AA$ found in trans-[Cr(NCS $\left.)_{2}(\mathrm{en})_{2}\right]_{2} \mathrm{ZnCl}_{4}$ (Moon \&
Table 1

Hydrogen-bond geometry $\left(\AA{ }^{\circ}\right)$.

\begin{tabular}{lllll}
\hline$D-\mathrm{H} \cdots A$ & $D-\mathrm{H}$ & $\mathrm{H} \cdots A$ & $D \cdots A$ & $D-\mathrm{H} \cdots A$ \\
\hline $\mathrm{N} 2 A-\mathrm{H} 2 A 1 \cdots \mathrm{S} 1^{\mathrm{i}}$ & 0.89 & 2.45 & $3.324(17)$ & 167 \\
$\mathrm{~N} 2 A-\mathrm{H} 2 A 2 \cdots \mathrm{O} 2 B^{\text {ii }}$ & 0.89 & 2.41 & $3.187(19)$ & 146 \\
$\mathrm{~N} 3 A-\mathrm{H} 3 A 1 \cdots \mathrm{O} 1 B^{\text {iii }}$ & 0.89 & 2.58 & $3.282(16)$ & 136 \\
$\mathrm{~N} 2 B-\mathrm{H} 2 B 1 \cdots \mathrm{S} 1^{\mathrm{i}}$ & 0.89 & 2.77 & $3.459(17)$ & 135 \\
$\mathrm{~N} 3 B-\mathrm{H} 3 B 1 \cdots \mathrm{O} 2 C^{\text {iii }}$ & 0.89 & 2.45 & $3.22(2)$ & 145 \\
$\mathrm{~N} 3 B-\mathrm{H} 3 B 2 \cdots \mathrm{S}^{\text {iv }}$ & 0.89 & 2.38 & $3.255(18)$ & 166 \\
\hline
\end{tabular}

Symmetry codes: (i) $-x+\frac{1}{2}, y-\frac{1}{2},-z+\frac{3}{2}$; (ii) $x, y-1, z$; (iii) $x,-y+1, z-\frac{1}{2}$; (iv) $-x+\frac{1}{2},-y+\frac{3}{2},-z+1$

Choi, 2015), trans- $\left[\mathrm{Cr}(\mathrm{NCS})_{2}(\text { cyclam })\right]_{2} \mathrm{ZnCl}_{4} \quad($ cyclam $=$ 1,4,8,11-tetraazacyclotetradecane (Moon et al., 2015), trans$\left[\mathrm{Cr}(\mathrm{NCS})_{2}\left(\mathrm{Me}_{2} \mathrm{tn}\right)_{2}\right] \mathrm{NCS}$ (Choi \& Lee, 2009) and cis$\left[\mathrm{Cr}(\mathrm{NCS})_{2}\right.$ (cyclam)]NCS (Moon et al., 2013), respectively. The $\mathrm{N}$-coordinated isothiocyanate group is almost linear, with an $\mathrm{N}-\mathrm{C}-\mathrm{S}$ angle of $179.3(3)^{\circ}$. The $\mathrm{ClO}_{4}{ }^{-}$counter-anion lies well outside the coordination sphere of the complex and, because of significant disorder, the tetrahedral geometry of this anion is severely distorted.

\section{Supramolecular features}

In the crystal, an $\mathrm{N}-\mathrm{H} \cdots \mathrm{S}$ hydrogen bond links neighbouring cations, while a series of $\mathrm{N}-\mathrm{H} \cdots \mathrm{O}$ contacts link the cations to neighbouring anions (Table 1). An extensive array of these contacts generate a three-dimensional network of molecules stacked along the $b$-axis direction (Fig. 2). These hydrogenbonded networks help to stabilize the crystal structure.

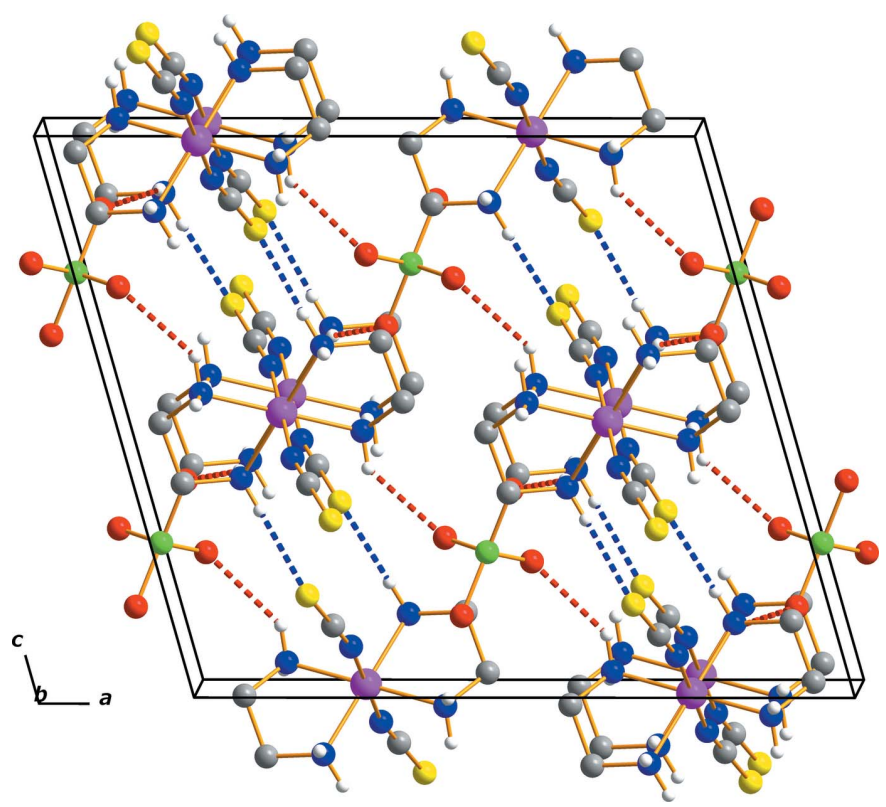

Figure 2

The crystal packing of (I), viewed perpendicular to the $a c$ plane. Dashed lines represent $\mathrm{N}-\mathrm{H} \cdots \mathrm{O}$ (red) and $\mathrm{N}-\mathrm{H} \cdots \mathrm{S}$ (blue) hydrogen-bonding interactions, respectively. The minor disorder components and C-bound $\mathrm{H}$ atoms have been omitted for clarity. 
Table 2

Experimental details.

\begin{tabular}{ll}
\hline Crystal data & \\
Chemical formula & {$\left[\mathrm{Cr}(\mathrm{NCS})_{2}\left(\mathrm{C}_{2} \mathrm{H}_{8} \mathrm{~N}_{2}\right)_{2}\right] \mathrm{ClO}_{4}$} \\
$M_{\mathrm{r}}$ & 387.82 \\
Crystal system, space group & Monoclinic, $C 2 / c$ \\
Temperature $(\mathrm{K})$ & 260 \\
$a, b, c(\AA)$ & $15.599(3), 7.4440(15), 13.792(3)$ \\
$\beta\left({ }^{\circ}\right)$ & $105.83(3)$ \\
$V\left(\AA^{3}\right)$ & $1540.8(6)$ \\
$Z$ & 4 \\
Radiation type & Synchrotron, $\lambda=0.630 \AA$ \\
$\mu\left(\mathrm{mm}^{-1}\right)$ & 0.86 \\
Crystal size $(\mathrm{mm})$ & $0.14 \times 0.13 \times 0.13$ \\
& \\
Data collection & ADSC Q210 CCD area detector \\
Diffractometer & Empirical (using intensity \\
Absorption correction & measurements) $(H K L 3000$ sm \\
& $S C A L E A P C K$; Otwinowski \& \\
& Minor, 1997$)$ \\
$T_{\text {min }}, T_{\text {max }}$ & $0.893,0.897$ \\
No. of measured, independent and & $8172,2121,2019$ \\
$\quad$ observed $[I>2 \sigma(I)]$ reflections & \\
$R_{\text {int }}$ & 0.015 \\
$(\text { sin } \theta / \lambda)_{\text {max }}\left(\AA^{-1}\right)$ & 0.696 \\
Refinement & \\
$R\left[F^{2}>2 \sigma\left(F^{2}\right)\right], w R\left(F^{2}\right), S$ & $0.060,0.178,1.09$ \\
No. of reflections & 2121 \\
No. of parameters & 140 \\
$H$-atom treatment & H-atom parameters constrained \\
$\Delta \rho_{\text {max }}, \Delta \rho_{\text {min }}\left(\mathrm{e} \AA^{-3}\right)$ & $0.74,-1.12$ \\
\hline
\end{tabular}

Computer programs: PAL ADSC Quantum-210 ADX Program (Arvai \& Nielsen, 1983), HKL3000sm (Otwinowski \& Minor, 1997), SHELXT2014/5 (Sheldrick, 2015a), SHELXL2014/7 (Sheldrick, 2015b), DIAMOND (Putz \& Brandenburg, 2014) and publCIF (Westrip, 2010).

\section{Database survey}

A search of the Cambridge Structural Database (Version 5.36, last update February 2015; Groom \& Allen, 2014) indicates a total of 13 hits for $\mathrm{Cr}^{\mathrm{III}}$ complexes with a $\left[\mathrm{Cr} L_{2}(\mathrm{en})_{2}\right]^{+}$unit. The crystal structures of trans- $\left[\mathrm{CrCl}_{2}(\mathrm{en})_{2}\right] \mathrm{Cl} \cdot \mathrm{HCl} \cdot 2 \mathrm{H}_{2} \mathrm{O}(\mathrm{Ooi}$ et al., 1960), trans- $\left[\mathrm{CrF}_{2}(\mathrm{en})_{2}\right] X\left(X=\mathrm{ClO}_{4}, \mathrm{Cl}, \mathrm{Br}\right)(\mathrm{Brenčič} \mathrm{\&}$ Leban, 1981), cis- $\left[\mathrm{CrF}_{2}(\mathrm{en})_{2}\right] \mathrm{ClO}_{4}$ (Brenčič et al., 1987), trans$\left[\mathrm{CrBr}_{2}(\text { en })_{2}\right] \mathrm{ClO}_{4}$ (Choi et al., 2010) have been reported previously. Recently, we have also reported the closely related crystal structure of $\left[\mathrm{Cr}(\mathrm{NCS})_{2}(\mathrm{en})_{2}\right]_{2}\left[\mathrm{ZnCl}_{4}\right]$, in which there are four crystallographically independent $\mathrm{Cr}^{\mathrm{III}}$ complex cations that also adopt a trans configuration. However, a crystal structure of $\left[\mathrm{Cr}(\mathrm{NCS})_{2}(\mathrm{en})_{2}\right]^{+}$with a $\mathrm{ClO}_{4}$ anion has not been reported previously.

\section{Synthesis and crystallization}

All chemicals were reagent grade materials and were used without further purification. The title compound, trans$\left[\mathrm{Cr}(\mathrm{NCS})_{2}(\mathrm{en})_{2}\right] \mathrm{ClO}_{4}$ was prepared according to the literature method (Sandrini et al., 1978). The crude perchlorate salt $(0.33 \mathrm{~g})$ was dissolved in $20 \mathrm{~mL}$ of $0.1 \mathrm{M} \mathrm{HCl}$ at $333 \mathrm{~K}$. The filtrate was added to $6 \mathrm{~mL}$ of $60 \% \mathrm{HClO}_{4}$. The resulting solution was allowed to stand at room temperature for $2 \mathrm{~d}$ to give orange block-like crystals suitable for X-ray structural analysis. IR spectrum (KBr, cm $\left.{ }^{-1}\right): 3247$ (vs), $3208(v s), 3131$ (vs) and $3097(v s)(v \mathrm{NH}), 2966(s), 2955(s)$ and $2893(s)(v$ $\mathrm{CH}), 2077$ (vs) ( $\left.v_{a} \mathrm{CN}\right), 1586(v s)\left(\delta \mathrm{NH}_{2}\right), 1459(s)\left(\delta \mathrm{CH}_{2}\right)$, $1365(m)(v \mathrm{CN}), 1326(s)\left(\omega \mathrm{NH}_{2}\right), 1290(v s)\left(\omega \mathrm{CH}_{2}\right), 1146$ $(v s)\left(\gamma \mathrm{NH}_{2}\right), 1117$ (vs) (v CN), $1088(v s)\left(v_{a} \mathrm{Cl}-\mathrm{O}\right), 1047$ (vs) $\left(\gamma \mathrm{CH}_{2}\right), 1007(s), 983(s), 873(m)\left(\rho \mathrm{CH}_{2}\right), 849(w)\left(\rho \mathrm{NH}_{2}\right)$, $729(v s), 636(s)$ and $626(v s)(\delta$ OClO), $558(v s), 559(s)$ $(\delta$ CCC), $501(v s), 478(s)$ ( $\delta$ NCS $), 444(m)$ and $419(m)(v$ $\mathrm{Cr}-\mathrm{N})$.

\section{Refinement}

Crystal data, data collection and structure refinement details are summarized in Table 2. In the title compound, the ethane1,2-diamine group is disordered with atoms $\mathrm{N} 2 A / \mathrm{N} 2 B, \mathrm{C} 2 A$ / $\mathrm{C} 2 B, \mathrm{C} 3 A / \mathrm{C} 3 B$ and $\mathrm{N} 3 A / \mathrm{N} 3 B$ positionally disordered over two sets of sites with a refined occupancy ratio of 0.522 (16):0.478 (16). The half molecules of each distorted perchlorate anion are disordered over two sites of equal occupancy, with atoms $\mathrm{Cl} 1 B / \mathrm{Cl} C \mathrm{C}$ and $\mathrm{O} 2 B / \mathrm{O} 1 C$ refined using EXYZ/EADP constraints. All $\mathrm{H}$ atoms were placed in geometrically idealized positions and constrained to ride on their parent atoms, with $\mathrm{C}-\mathrm{H}=0.97 \AA$ and $\mathrm{N}-\mathrm{H}=0.89 \AA$, and with $U_{\text {iso }}(\mathrm{H})$ values of 1.2 of the parent atoms.

\section{Acknowledgements}

The X-ray crystallography experiment at the PLS-II BL2DSMC beamline was supported in part by MISP and POSTECH.

\section{References}

Arvai, A. J. \& Nielsen, C. (1983). ADSC Quantum-210 ADX. Area Detector System Corporation, Poway, CA, USA.

Brenčič, J. V. \& Leban, I. (1981). Z. Anorg. Allg. Chem. 480, 213-219. Brenčič, J. V., Leban, I. \& Polanc, I. (1987). Acta Cryst. C43, 885-887. Choi, J.-H. (2000). Chem. Phys. 256, 29-35.

Choi, J.-H., Clegg, W., Harrington, R. W. \& Lee, S. H. (2010). J. Chem. Crystallogr. 40, 567-571.

Choi, J.-H., Joshi, T. \& Spiccia, L. (2011). Z. Anorg. Allg. Chem. 637, 1194-1198.

Choi, J.-H. \& Lee, S. H. (2009). J. Mol. Struct. 932, 84-89.

Choi, J.-H. \& Moon, D. (2014). J. Mol. Struct. 1059, 325-331.

Choi, J.-H., Oh, I. G., Suzuki, T. \& Kaizaki, S. (2004). J. Mol. Struct. 694, 39-44.

De, G., Szuki, M. \& Uehara, A. (1987). Bull. Chem. Soc. Jpn, 60, 28712874.

Groom, C. R. \& Allen, F. H. (2014). Angew. Chem. Int. Ed. 35, 31033111.

House, D. A. (1973). J. Inorg. Nucl. Chem. 53, 662-671.

Moon, D. \& Choi, J.-H. (2015). Acta Cryst. E71, 100-103.

Moon, D., Choi, J.-H., Ryoo, K. S. \& Hong, Y. P. (2013). Acta Cryst. E69, m376-m377.

Moon, D., Ryoo, K. S. \& Choi, J.-H. (2015). Acta Cryst. E71, 540-543.

Ooi, S., Komiyama, Y. \& Kuroya, H. (1960). Bull. Chem. Soc. Jpn, 33, 354-357.

Otwinowski, Z. \& Minor, W. (1997). Methods in Enzymology, Vol. 276, Macromolecular Crystallography, Part A, edited by C. W. Carter Jr \& R. M. Sweet, pp. 307-326. New York: Academic Press. 
Putz, H. \& Brandenburg, K. (2014). DIAMOND. Crystal Impact GbR, Bonn, Germany.

Sandrini, D., Gandolfi, M. T., Moggi, L. \& Balzani, V. (1978). J. Am. Chem. Soc. 100, 1463-1468.
Sheldrick, G. M. (2015a). Acta Cryst. A71, 3-8. Sheldrick, G. M. (2015b). Acta Cryst. C71, 3-8.

Westrip, S. P. (2010). J. Appl. Cryst. 43, 920-925. 


\section{supporting information}

Acta Cryst. (2015). E71, 650-653 [doi:10.1107/S2056989015009184]

\section{Crystal structure of trans-bis(ethane-1,2-diamine- $\left.\kappa^{2} N, N^{\prime}\right)$ bis(thiocyanato- $\kappa N)$ chromium(III) perchlorate from synchrotron data}

\section{Dohyun Moon and Jong-Ha Choi}

\section{Computing details}

Data collection: PAL ADSC Quantum-210 ADX Program (Arvai \& Nielsen, 1983); cell refinement: HKL3000sm (Otwinowski \& Minor, 1997); data reduction: HKL3000sm (Otwinowski \& Minor,1997); program(s) used to solve structure: SHELXT2014/5 (Sheldrick, 2015a); program(s) used to refine structure: SHELXL2014/7 (Sheldrick, 2015b); molecular graphics: DIAMOND (Putz \& Brandenburg, 2014); software used to prepare material for publication: publCIF (Westrip, 2010).

\section{trans-Bis(ethane-1,2-diamine- $\left.\kappa^{2} N, N^{\prime}\right)$ bis(thiocyanato- $\left.\kappa N\right)$ chromium(III) perchlorate}

Crystal data

$\left[\mathrm{Cr}(\mathrm{NCS})_{2}\left(\mathrm{C}_{2} \mathrm{H}_{8} \mathrm{~N}_{2}\right)_{2}\right] \mathrm{ClO}_{4}$

$M_{r}=387.82$

Monoclinic, $C 2 / c$

$a=15.599(3) \AA$

$b=7.4440(15) \AA$

$c=13.792(3) \AA$

$\beta=105.83(3)^{\circ}$

$V=1540.8(6) \AA^{3}$

$Z=4$

$F(000)=796$

$D_{\mathrm{x}}=1.672 \mathrm{Mg} \mathrm{m}^{-3}$

Synchrotron radiation, $\lambda=0.630 \AA$

Cell parameters from 46962 reflections

$\theta=0.4-33.6^{\circ}$

$\mu=0.86 \mathrm{~mm}^{-1}$

$T=260 \mathrm{~K}$

Block, orange

$0.14 \times 0.13 \times 0.13 \mathrm{~mm}$

Data collection

ADSC Q210 CCD area-detector diffractometer

Radiation source: PLSII 2D bending magnet

$\omega$ scan

Absorption correction: empirical (using intensity measurements)

(HKL3000sm SCALEAPCK; Otwinowski \& Minor, 1997)

$T_{\min }=0.893, T_{\max }=0.897$

\section{Refinement}

Refinement on $F^{2}$

Least-squares matrix: full

$R\left[F^{2}>2 \sigma\left(F^{2}\right)\right]=0.060$

$w R\left(F^{2}\right)=0.178$

$S=1.09$

2121 reflections

140 parameters

0 restraints
8172 measured reflections

2121 independent reflections

2019 reflections with $I>2 \sigma(I)$

$R_{\text {int }}=0.015$

$\theta_{\text {max }}=26.0^{\circ}, \theta_{\min }=2.7^{\circ}$

$h=-21 \rightarrow 21$

$k=-10 \rightarrow 10$

$l=-19 \rightarrow 19$

Hydrogen site location: inferred from neighbouring sites

$\mathrm{H}$-atom parameters constrained

$w=1 /\left[\sigma^{2}\left(F_{\mathrm{o}}^{2}\right)+(0.1146 P)^{2}+2.4721 P\right]$

where $P=\left(F_{\mathrm{o}}^{2}+2 F_{\mathrm{c}}^{2}\right) / 3$

$(\Delta / \sigma)_{\max }<0.001$

$\Delta \rho_{\max }=0.74 \mathrm{e} \AA^{-3}$

$\Delta \rho_{\min }=-1.12 \mathrm{e}^{-3}$ 
Extinction correction: SHELXL2014/7

(Sheldrick, 2015b),

$\mathrm{Fc}^{*}=\mathrm{kFc}\left[1+0.001 \times \mathrm{xc}^{2} \lambda^{3} / \sin (2 \theta)\right]^{-1 / 4}$

Extinction coefficient: 0.045 (12)

Special details

Geometry. All e.s.d.'s (except the e.s.d. in the dihedral angle between two 1.s. planes) are estimated using the full covariance matrix. The cell e.s.d.'s are taken into account individually in the estimation of e.s.d.'s in distances, angles and torsion angles; correlations between e.s.d.'s in cell parameters are only used when they are defined by crystal symmetry. An approximate (isotropic) treatment of cell e.s.d.'s is used for estimating e.s.d.'s involving l.s. planes.

Fractional atomic coordinates and isotropic or equivalent isotropic displacement parameters $\left(\AA^{2}\right)$

\begin{tabular}{|c|c|c|c|c|c|}
\hline & $x$ & $y$ & $z$ & $U_{\text {iso }} * / U_{\text {eq }}$ & Occ. $(<1)$ \\
\hline $\mathrm{Cr} 1$ & 0.2500 & 0.2500 & 0.5000 & $0.0273(3)$ & \\
\hline S1 & $0.21080(8)$ & $0.77661(11)$ & $0.67477(8)$ & $0.0586(3)$ & \\
\hline $\mathrm{N} 1$ & $0.24831(15)$ & $0.4775(3)$ & $0.57426(17)$ & $0.0433(5)$ & \\
\hline $\mathrm{C} 1$ & $0.23290(16)$ & $0.6026(3)$ & $0.61573(17)$ & $0.0363(5)$ & \\
\hline $\mathrm{N} 2 \mathrm{~A}$ & $0.3423(12)$ & $0.1308(19)$ & $0.6213(13)$ & $0.036(2)$ & $0.522(16)$ \\
\hline $\mathrm{H} 2 \mathrm{~A} 1$ & 0.3272 & 0.1503 & 0.6781 & $0.043^{*}$ & $0.522(16)$ \\
\hline $\mathrm{H} 2 \mathrm{~A} 2$ & 0.3442 & 0.0127 & 0.6118 & $0.043^{*}$ & $0.522(16)$ \\
\hline N3A & $0.3624(11)$ & $0.337(2)$ & $0.4641(10)$ & $0.043(3)$ & $0.522(16)$ \\
\hline H3A1 & 0.3553 & 0.3266 & 0.3981 & $0.052^{*}$ & $0.522(16)$ \\
\hline $\mathrm{H} 3 \mathrm{~A} 2$ & 0.3722 & 0.4525 & 0.4807 & $0.052 *$ & $0.522(16)$ \\
\hline $\mathrm{C} 2 \mathrm{~A}$ & $0.4311(5)$ & $0.2126(10)$ & $0.6277(8)$ & $0.057(2)$ & $0.522(16)$ \\
\hline $\mathrm{H} 2 \mathrm{~A} 3$ & 0.4784 & 0.1375 & 0.6678 & $0.068^{*}$ & $0.522(16)$ \\
\hline $\mathrm{H} 2 \mathrm{~A} 4$ & 0.4355 & 0.3305 & 0.6587 & $0.068^{*}$ & $0.522(16)$ \\
\hline $\mathrm{C} 3 \mathrm{~A}$ & $0.4385(5)$ & $0.2274(14)$ & $0.5199(10)$ & $0.066(3)$ & $0.522(16)$ \\
\hline H3A3 & 0.4943 & 0.2842 & 0.5191 & $0.079^{*}$ & $0.522(16)$ \\
\hline $\mathrm{H} 3 \mathrm{~A} 4$ & 0.4362 & 0.1092 & 0.4897 & $0.079 *$ & $0.522(16)$ \\
\hline $\mathrm{N} 2 \mathrm{~B}$ & $0.3570(13)$ & $0.164(2)$ & $0.6143(14)$ & $0.041(3)$ & $0.478(16)$ \\
\hline H2B1 & 0.3654 & 0.2382 & 0.6667 & $0.049^{*}$ & $0.478(16)$ \\
\hline $\mathrm{H} 2 \mathrm{~B} 2$ & 0.3464 & 0.0548 & 0.6342 & $0.049 *$ & $0.478(16)$ \\
\hline N3B & $0.3502(13)$ & $0.341(3)$ & $0.4378(9)$ & $0.040(2)$ & $0.478(16)$ \\
\hline H3B1 & 0.3527 & 0.2732 & 0.3856 & $0.048^{*}$ & $0.478(16)$ \\
\hline H3B2 & 0.3396 & 0.4543 & 0.4167 & $0.048^{*}$ & $0.478(16)$ \\
\hline $\mathrm{C} 2 \mathrm{~B}$ & $0.4369(4)$ & $0.1614(15)$ & $0.5773(8)$ & $0.056(2)$ & $0.478(16)$ \\
\hline H2B3 & 0.4355 & 0.0582 & 0.5339 & $0.067^{*}$ & 0.478 (16) \\
\hline H2B4 & 0.4902 & 0.1541 & 0.6334 & $0.067^{*}$ & $0.478(16)$ \\
\hline $\mathrm{C} 3 \mathrm{~B}$ & $0.4370(5)$ & $0.3297(19)$ & $0.5203(7)$ & 0.060 & 0.478 (16) \\
\hline H3B3 & 0.4869 & 0.3303 & 0.4911 & $0.072^{*}$ & $0.478(16)$ \\
\hline H3B4 & 0.4427 & 0.4322 & 0.5651 & $0.072 *$ & $0.478(16)$ \\
\hline Cl1B & 0.5000 & $0.7072(3)$ & 0.7500 & $0.0989(7)$ & 0.5 \\
\hline O1B & 0.4393 & $0.5672(9)$ & $0.7711(5)$ & $0.0762(16)$ & 0.5 \\
\hline $\mathrm{O} 2 \mathrm{~B}$ & $0.4350(6)$ & $0.7462(8)$ & $0.6376(6)$ & $0.159(3)$ & 0.5 \\
\hline $\mathrm{Cl1C}$ & 0.5000 & $0.7072(3)$ & 0.7500 & $0.0989(7)$ & 0.5 \\
\hline $\mathrm{O} 1 \mathrm{C}$ & $0.4350(6)$ & $0.7462(8)$ & $0.6376(6)$ & $0.159(3)$ & 0.5 \\
\hline $\mathrm{O} 2 \mathrm{C}$ & $0.4488(11)$ & $0.8416(15)$ & $0.7860(8)$ & $0.152(5)$ & 0.5 \\
\hline
\end{tabular}


Atomic displacement parameters $\left(\AA^{2}\right)$

\begin{tabular}{lllllll}
\hline & $U^{11}$ & $U^{22}$ & $U^{33}$ & $U^{12}$ & $U^{13}$ & $U^{23}$ \\
\hline $\mathrm{Cr} 1$ & $0.0366(4)$ & $0.0229(3)$ & $0.0251(3)$ & $0.00353(14)$ & $0.0128(2)$ & $-0.00135(14)$ \\
$\mathrm{S} 1$ & $0.0988(7)$ & $0.0298(4)$ & $0.0630(6)$ & $0.0072(3)$ & $0.0492(5)$ & $-0.0070(3)$ \\
$\mathrm{N} 1$ & $0.0553(12)$ & $0.0327(11)$ & $0.0427(10)$ & $0.0052(9)$ & $0.0145(9)$ & $-0.0097(8)$ \\
$\mathrm{C} 1$ & $0.0472(12)$ & $0.0289(10)$ & $0.0356(10)$ & $0.0012(9)$ & $0.0163(9)$ & $-0.0012(8)$ \\
$\mathrm{N} 2 \mathrm{~A}$ & $0.050(5)$ & $0.024(3)$ & $0.034(3)$ & $-0.001(2)$ & $0.011(3)$ & $0.004(2)$ \\
$\mathrm{N} 3 \mathrm{~A}$ & $0.047(5)$ & $0.036(3)$ & $0.056(7)$ & $0.006(3)$ & $0.028(5)$ & $0.017(5)$ \\
$\mathrm{C} 2 \mathrm{~A}$ & $0.048(3)$ & $0.044(3)$ & $0.065(5)$ & $-0.003(2)$ & $-0.006(3)$ & $-0.001(3)$ \\
$\mathrm{C} 3 \mathrm{~A}$ & $0.038(3)$ & $0.044(4)$ & $0.121(8)$ & $0.008(3)$ & $0.031(4)$ & $0.026(5)$ \\
$\mathrm{N} 2 \mathrm{~B}$ & $0.048(6)$ & $0.044(8)$ & $0.031(3)$ & $0.012(5)$ & $0.014(3)$ & $0.005(4)$ \\
$\mathrm{N} 3 \mathrm{~B}$ & $0.050(5)$ & $0.043(4)$ & $0.031(4)$ & $0.005(3)$ & $0.019(4)$ & $0.002(3)$ \\
$\mathrm{C} 2 \mathrm{~B}$ & $0.041(3)$ & $0.073(5)$ & $0.051(5)$ & $0.017(3)$ & $0.007(3)$ & $0.003(4)$ \\
$\mathrm{C} 3 \mathrm{~B}$ & $0.045(3)$ & $0.068(7)$ & $0.070(4)$ & $-0.014(4)$ & $0.023(3)$ & $-0.013(4)$ \\
$\mathrm{C} 11 \mathrm{~B}$ & $0.1112(15)$ & $0.0671(10)$ & $0.1316(18)$ & 0.000 & $0.0553(13)$ & 0.000 \\
O1B & $0.074(3)$ & $0.073(4)$ & $0.083(4)$ & $-0.009(3)$ & $0.024(3)$ & $0.029(3)$ \\
O2B & $0.152(6)$ & $0.199(8)$ & $0.130(5)$ & $0.028(4)$ & $0.045(5)$ & $0.035(4)$ \\
C11C & $0.1112(15)$ & $0.0671(10)$ & $0.1316(18)$ & 0.000 & $0.0553(13)$ & 0.000 \\
O1C & $0.152(6)$ & $0.199(8)$ & $0.130(5)$ & $0.028(4)$ & $0.045(5)$ & $0.035(4)$ \\
O2C & $0.255(15)$ & $0.095(7)$ & $0.121(8)$ & $-0.038(9)$ & $0.076(9)$ & $-0.014(6)$ \\
& & & & & &
\end{tabular}

Geometric parameters $\left(\AA,{ }^{\circ}\right)$

\begin{tabular}{llll}
\hline $\mathrm{Cr} 1-\mathrm{N} 1$ & $1.983(2)$ & $\mathrm{C} 3 \mathrm{~A}-\mathrm{H} 3 \mathrm{~A} 3$ & 0.9700 \\
$\mathrm{Cr} 1-\mathrm{N} 1^{\mathrm{i}}$ & $1.983(2)$ & $\mathrm{C} 3 \mathrm{~A}-\mathrm{H} 3 \mathrm{~A} 4$ & 0.9700 \\
$\mathrm{Cr} 1-\mathrm{N} 3 \mathrm{~A}^{\mathrm{i}}$ & $2.053(16)$ & $\mathrm{N} 2 \mathrm{~B}-\mathrm{C} 2 \mathrm{~B}$ & $1.471(18)$ \\
$\mathrm{Cr} 1-\mathrm{N} 3 \mathrm{~A}$ & $2.053(16)$ & $\mathrm{N} 2 \mathrm{~B}-\mathrm{H} 2 \mathrm{~B} 1$ & 0.8900 \\
$\mathrm{Cr} 1-\mathrm{N} 2 \mathrm{~B}$ & $2.06(2)$ & $\mathrm{N} 2 \mathrm{~B}-\mathrm{H} 2 \mathrm{~B} 2$ & 0.8900 \\
$\mathrm{Cr} 1-\mathrm{N} 2 \mathrm{~B}^{\mathrm{i}}$ & $2.06(2)$ & $\mathrm{N} 3 \mathrm{~B}-\mathrm{C} 3 \mathrm{~B}$ & $1.514(17)$ \\
$\mathrm{Cr} 1-\mathrm{N} 2 \mathrm{~A}^{\mathrm{i}}$ & $2.085(19)$ & $\mathrm{N} 3 \mathrm{~B}-\mathrm{H} 3 \mathrm{~B} 1$ & 0.8900 \\
$\mathrm{Cr} 1-\mathrm{N} 2 \mathrm{~A}$ & $2.085(19)$ & $\mathrm{N} 3 \mathrm{~B}-\mathrm{H} 3 \mathrm{~B} 2$ & 0.8900 \\
$\mathrm{Cr} 1-\mathrm{N} 3 \mathrm{~B}^{\mathrm{i}}$ & $2.09(2)$ & $\mathrm{C} 2 \mathrm{~B}-\mathrm{C} 3 \mathrm{~B}$ & $1.479(17)$ \\
$\mathrm{Cr} 1-\mathrm{N} 3 \mathrm{~B}$ & $2.09(2)$ & $\mathrm{C} 2 \mathrm{~B}-\mathrm{H} 2 \mathrm{~B} 3$ & 0.9700 \\
$\mathrm{~S} 1-\mathrm{C} 1$ & $1.617(3)$ & $\mathrm{C} 2 \mathrm{~B}-\mathrm{H} 2 \mathrm{~B} 4$ & 0.9700 \\
$\mathrm{~N} 1-\mathrm{C} 1$ & $1.152(3)$ & $\mathrm{C} 3 \mathrm{~B}-\mathrm{H} 3 \mathrm{~B} 3$ & 0.9700 \\
$\mathrm{~N} 2 \mathrm{~A}-\mathrm{C} 2 \mathrm{~A}$ & $1.493(15)$ & $\mathrm{C} 3 \mathrm{~B}-\mathrm{H} 3 \mathrm{~B} 4$ & 0.9700 \\
$\mathrm{~N} 2 \mathrm{~A}-\mathrm{H} 2 \mathrm{~A} 1$ & 0.8900 & $\mathrm{C} 11 \mathrm{~B}-\mathrm{O} 1 \mathrm{~B}^{\mathrm{ii}}$ & $1.489(6)$ \\
$\mathrm{N} 2 \mathrm{~A}-\mathrm{H} 2 \mathrm{~A} 2$ & 0.8900 & $\mathrm{Cl1B}-\mathrm{O} 1 \mathrm{~B}$ & $1.489(6)$ \\
$\mathrm{N} 3 \mathrm{~A}-\mathrm{C} 3 \mathrm{~A}$ & $1.475(16)$ & $\mathrm{C} 11 \mathrm{~B}-\mathrm{O} 2 \mathrm{~B}^{\mathrm{ii}}$ & $1.630(8)$ \\
$\mathrm{N} 3 \mathrm{~A}-\mathrm{H} 3 \mathrm{~A} 1$ & 0.8900 & $\mathrm{C} 11 \mathrm{~B}-\mathrm{O} 2 \mathrm{~B}$ & $1.630(8)$ \\
$\mathrm{N} 3 \mathrm{~A}-\mathrm{H} 3 \mathrm{~A} 2$ & 0.8900 & $\mathrm{C} 11 \mathrm{C}-\mathrm{O} 2 \mathrm{C}^{\mathrm{ii}}$ & $1.450(13)$ \\
$\mathrm{C} 2 \mathrm{~A}-\mathrm{C} 3 \mathrm{~A}$ & $1.527(17)$ & $\mathrm{C} 11 \mathrm{C}-\mathrm{O} 2 \mathrm{C}$ & $1.450(13)$ \\
$\mathrm{C} 2 \mathrm{~A}-\mathrm{H} 2 \mathrm{~A} 3$ & 0.9700 & $\mathrm{C} 11 \mathrm{C}-\mathrm{O} 1 \mathrm{C}^{\mathrm{ii}}$ & $1.630(8)$ \\
$\mathrm{C} 2 \mathrm{~A}-\mathrm{H} 2 \mathrm{~A} 4$ & $\mathrm{C} 11 \mathrm{C}-\mathrm{O} 1 \mathrm{C}$ & $1.630(8)$ \\
& 0.9700 & & 110.4 \\
$\mathrm{~N} 1-\mathrm{Cr} 1-\mathrm{N} 1^{\mathrm{i}}$ & & $\mathrm{N} 2 \mathrm{~A}-\mathrm{C} 2 \mathrm{~A}-\mathrm{H} 2 \mathrm{~A} 4$ & 110.4 \\
$\mathrm{~N} 1-\mathrm{Cr} 1-\mathrm{N} 3 \mathrm{~A}^{\mathrm{i}}$ & 180.0 & $\mathrm{C} 3 \mathrm{~A}-\mathrm{C} 2 \mathrm{~A}-\mathrm{H} 2 \mathrm{~A} 4$ & \\
& $90.8(5)$ & &
\end{tabular}




\begin{tabular}{|c|c|c|c|}
\hline $\mathrm{N} 1{ }^{\mathrm{i}}-\mathrm{Cr} 1-\mathrm{N} 3 \mathrm{~A}^{\mathrm{i}}$ & $89.2(5)$ & $\mathrm{H} 2 \mathrm{~A} 3-\mathrm{C} 2 \mathrm{~A}-\mathrm{H} 2 \mathrm{~A} 4$ & 108.6 \\
\hline $\mathrm{N} 1-\mathrm{Cr} 1-\mathrm{N} 3 \mathrm{~A}$ & $89.2(5)$ & $\mathrm{N} 3 \mathrm{~A}-\mathrm{C} 3 \mathrm{~A}-\mathrm{C} 2 \mathrm{~A}$ & $106.5(10)$ \\
\hline $\mathrm{N} 1-\mathrm{Cr} 1-\mathrm{N} 3 \mathrm{~A}$ & $90.8(5)$ & $\mathrm{N} 3 \mathrm{~A}-\mathrm{C} 3 \mathrm{~A}-\mathrm{H} 3 \mathrm{~A} 3$ & 110.4 \\
\hline $\mathrm{N} 3 \mathrm{~A}^{\mathrm{i}}-\mathrm{Cr} 1-\mathrm{N} 3 \mathrm{~A}$ & 180.0 & $\mathrm{C} 2 \mathrm{~A}-\mathrm{C} 3 \mathrm{~A}-\mathrm{H} 3 \mathrm{~A} 3$ & 110.4 \\
\hline $\mathrm{N} 1-\mathrm{Cr} 1-\mathrm{N} 2 \mathrm{~B}$ & $89.5(5)$ & $\mathrm{N} 3 \mathrm{~A}-\mathrm{C} 3 \mathrm{~A}-\mathrm{H} 3 \mathrm{~A} 4$ & 110.4 \\
\hline $\mathrm{N} 1{ }^{\mathrm{i}}-\mathrm{Cr} 1-\mathrm{N} 2 \mathrm{~B}$ & $90.5(5)$ & $\mathrm{C} 2 \mathrm{~A}-\mathrm{C} 3 \mathrm{~A}-\mathrm{H} 3 \mathrm{~A} 4$ & 110.4 \\
\hline $\mathrm{N} 1-\mathrm{Cr} 1-\mathrm{N} 2 \mathrm{~B}^{\mathrm{i}}$ & $90.5(5)$ & $\mathrm{H} 3 \mathrm{~A} 3-\mathrm{C} 3 \mathrm{~A}-\mathrm{H} 3 \mathrm{~A} 4$ & 108.6 \\
\hline $\mathrm{N} 1{ }^{\mathrm{i}}-\mathrm{Cr} 1-\mathrm{N} 2 \mathrm{~B}^{\mathrm{i}}$ & $89.5(5)$ & $\mathrm{C} 2 \mathrm{~B}-\mathrm{N} 2 \mathrm{~B}-\mathrm{Cr} 1$ & $109.0(9)$ \\
\hline $\mathrm{N} 2 \mathrm{~B}-\mathrm{Cr} 1-\mathrm{N} 2 \mathrm{~B}^{\mathrm{i}}$ & $180.0(9)$ & $\mathrm{C} 2 \mathrm{~B}-\mathrm{N} 2 \mathrm{~B}-\mathrm{H} 2 \mathrm{~B} 1$ & 109.9 \\
\hline $\mathrm{N} 1-\mathrm{Cr} 1-\mathrm{N} 2 \mathrm{~A}^{\mathrm{i}}$ & $87.0(4)$ & $\mathrm{Cr} 1-\mathrm{N} 2 \mathrm{~B}-\mathrm{H} 2 \mathrm{~B} 1$ & 109.9 \\
\hline $\mathrm{N} 1{ }^{\mathrm{i}}-\mathrm{Cr} 1-\mathrm{N} 2 \mathrm{~A}^{\mathrm{i}}$ & $93.0(5)$ & $\mathrm{C} 2 \mathrm{~B}-\mathrm{N} 2 \mathrm{~B}-\mathrm{H} 2 \mathrm{~B} 2$ & 109.9 \\
\hline $\mathrm{N} 3 \mathrm{~A}^{\mathrm{i}}-\mathrm{Cr} 1-\mathrm{N} 2 \mathrm{~A}^{\mathrm{i}}$ & $83.1(5)$ & $\mathrm{Cr} 1-\mathrm{N} 2 \mathrm{~B}-\mathrm{H} 2 \mathrm{~B} 2$ & 109.9 \\
\hline $\mathrm{N} 3 \mathrm{~A}-\mathrm{Cr} 1-\mathrm{N} 2 \mathrm{~A}^{\mathrm{i}}$ & $96.9(5)$ & $\mathrm{H} 2 \mathrm{~B} 1-\mathrm{N} 2 \mathrm{~B}-\mathrm{H} 2 \mathrm{~B} 2$ & 108.3 \\
\hline $\mathrm{N} 1-\mathrm{Cr} 1-\mathrm{N} 2 \mathrm{~A}$ & $93.0(4)$ & $\mathrm{C} 3 \mathrm{~B}-\mathrm{N} 3 \mathrm{~B}-\mathrm{Cr} 1$ & $106.7(8)$ \\
\hline $\mathrm{N} 1-\mathrm{Cr} 1-\mathrm{N} 2 \mathrm{~A}$ & $87.0(4)$ & $\mathrm{C} 3 \mathrm{~B}-\mathrm{N} 3 \mathrm{~B}-\mathrm{H} 3 \mathrm{~B} 1$ & 110.4 \\
\hline $\mathrm{N} 3 \mathrm{~A}^{\mathrm{i}}-\mathrm{Cr} 1-\mathrm{N} 2 \mathrm{~A}$ & $96.9(5)$ & $\mathrm{Cr} 1-\mathrm{N} 3 \mathrm{~B}-\mathrm{H} 3 \mathrm{~B} 1$ & 110.4 \\
\hline $\mathrm{N} 3 \mathrm{~A}-\mathrm{Cr} 1-\mathrm{N} 2 \mathrm{~A}$ & $83.1(5)$ & $\mathrm{C} 3 \mathrm{~B}-\mathrm{N} 3 \mathrm{~B}-\mathrm{H} 3 \mathrm{~B} 2$ & 110.4 \\
\hline $\mathrm{N} 2 \mathrm{~A}-\mathrm{Cr} 1-\mathrm{N} 2 \mathrm{~A}$ & 180.0 & $\mathrm{Cr} 1-\mathrm{N} 3 \mathrm{~B}-\mathrm{H} 3 \mathrm{~B} 2$ & 110.4 \\
\hline $\mathrm{N} 1-\mathrm{Cr} 1-\mathrm{N} 3 \mathrm{~B}^{\mathrm{i}}$ & $87.1(5)$ & $\mathrm{H} 3 \mathrm{~B} 1-\mathrm{N} 3 \mathrm{~B}-\mathrm{H} 3 \mathrm{~B} 2$ & 108.6 \\
\hline $\mathrm{N} 1-\mathrm{Cr} 1-\mathrm{N} 3 \mathrm{~B}^{\mathrm{i}}$ & $92.9(5)$ & $\mathrm{N} 2 \mathrm{~B}-\mathrm{C} 2 \mathrm{~B}-\mathrm{C} 3 \mathrm{~B}$ & $107.1(10)$ \\
\hline $\mathrm{N} 2 \mathrm{~B}-\mathrm{Cr} 1-\mathrm{N} 3 \mathrm{~B}^{\mathrm{i}}$ & $97.2(5)$ & $\mathrm{N} 2 \mathrm{~B}-\mathrm{C} 2 \mathrm{~B}-\mathrm{H} 2 \mathrm{~B} 3$ & 110.3 \\
\hline $\mathrm{N} 2 \mathrm{~B}^{\mathrm{i}}-\mathrm{Cr} 1-\mathrm{N} 3 \mathrm{~B}^{\mathrm{i}}$ & $82.8(5)$ & $\mathrm{C} 3 \mathrm{~B}-\mathrm{C} 2 \mathrm{~B}-\mathrm{H} 2 \mathrm{~B} 3$ & 110.3 \\
\hline $\mathrm{N} 1-\mathrm{Cr} 1-\mathrm{N} 3 \mathrm{~B}$ & $92.9(5)$ & $\mathrm{N} 2 \mathrm{~B}-\mathrm{C} 2 \mathrm{~B}-\mathrm{H} 2 \mathrm{~B} 4$ & 110.3 \\
\hline $\mathrm{N} 1-\mathrm{Cr} 1-\mathrm{N} 3 \mathrm{~B}$ & $87.1(5)$ & $\mathrm{C} 3 \mathrm{~B}-\mathrm{C} 2 \mathrm{~B}-\mathrm{H} 2 \mathrm{~B} 4$ & 110.3 \\
\hline $\mathrm{N} 2 \mathrm{~B}-\mathrm{Cr} 1-\mathrm{N} 3 \mathrm{~B}$ & $82.8(5)$ & $\mathrm{H} 2 \mathrm{~B} 3-\mathrm{C} 2 \mathrm{~B}-\mathrm{H} 2 \mathrm{~B} 4$ & 108.5 \\
\hline $\mathrm{N} 2 \mathrm{~B}-\mathrm{Cr} 1-\mathrm{N} 3 \mathrm{~B}$ & $97.2(5)$ & $\mathrm{C} 2 \mathrm{~B}-\mathrm{C} 3 \mathrm{~B}-\mathrm{N} 3 \mathrm{~B}$ & $108.6(10)$ \\
\hline $\mathrm{N} 3 \mathrm{~B}^{\mathrm{i}}-\mathrm{Cr} 1-\mathrm{N} 3 \mathrm{~B}$ & 180.0 & $\mathrm{C} 2 \mathrm{~B}-\mathrm{C} 3 \mathrm{~B}-\mathrm{H} 3 \mathrm{~B} 3$ & 110.0 \\
\hline $\mathrm{C} 1-\mathrm{N} 1-\mathrm{Cr} 1$ & $168.7(2)$ & $\mathrm{N} 3 \mathrm{~B}-\mathrm{C} 3 \mathrm{~B}-\mathrm{H} 3 \mathrm{~B} 3$ & 110.0 \\
\hline $\mathrm{N} 1-\mathrm{C} 1-\mathrm{S} 1$ & $179.3(3)$ & $\mathrm{C} 2 \mathrm{~B}-\mathrm{C} 3 \mathrm{~B}-\mathrm{H} 3 \mathrm{~B} 4$ & 110.0 \\
\hline $\mathrm{C} 2 \mathrm{~A}-\mathrm{N} 2 \mathrm{~A}-\mathrm{Cr} 1$ & $107.5(7)$ & $\mathrm{N} 3 \mathrm{~B}-\mathrm{C} 3 \mathrm{~B}-\mathrm{H} 3 \mathrm{~B} 4$ & 110.0 \\
\hline $\mathrm{C} 2 \mathrm{~A}-\mathrm{N} 2 \mathrm{~A}-\mathrm{H} 2 \mathrm{~A} 1$ & 110.2 & $\mathrm{H} 3 \mathrm{~B} 3-\mathrm{C} 3 \mathrm{~B}-\mathrm{H} 3 \mathrm{~B} 4$ & 108.4 \\
\hline $\mathrm{Cr} 1-\mathrm{N} 2 \mathrm{~A}-\mathrm{H} 2 \mathrm{~A} 1$ & 110.2 & $\mathrm{O} 1 \mathrm{~B}^{\mathrm{ii}}-\mathrm{C} 11 \mathrm{~B}-\mathrm{O} 1 \mathrm{~B}$ & $91.2(5)$ \\
\hline $\mathrm{C} 2 \mathrm{~A}-\mathrm{N} 2 \mathrm{~A}-\mathrm{H} 2 \mathrm{~A} 2$ & 110.2 & $\mathrm{O} 1 \mathrm{~B}^{\mathrm{ii}}-\mathrm{C} 11 \mathrm{~B}-\mathrm{O} 2 \mathrm{~B}^{\mathrm{ii}}$ & $92.7(4)$ \\
\hline $\mathrm{Cr} 1-\mathrm{N} 2 \mathrm{~A}-\mathrm{H} 2 \mathrm{~A} 2$ & 110.2 & $\mathrm{O} 1 \mathrm{~B}-\mathrm{C} 11 \mathrm{~B}-\mathrm{O} 2 \mathrm{~B}^{\mathrm{ii}}$ & $101.7(3)$ \\
\hline $\mathrm{H} 2 \mathrm{~A} 1-\mathrm{N} 2 \mathrm{~A}-\mathrm{H} 2 \mathrm{~A} 2$ & 108.5 & $\mathrm{O} 1 \mathrm{~B}^{\mathrm{ii}}-\mathrm{C} 11 \mathrm{~B}-\mathrm{O} 2 \mathrm{~B}$ & $101.7(3)$ \\
\hline $\mathrm{C} 3 \mathrm{~A}-\mathrm{N} 3 \mathrm{~A}-\mathrm{Cr} 1$ & $108.5(8)$ & $\mathrm{O} 1 \mathrm{~B}-\mathrm{C} 11 \mathrm{~B}-\mathrm{O} 2 \mathrm{~B}$ & $92.7(4)$ \\
\hline $\mathrm{C} 3 \mathrm{~A}-\mathrm{N} 3 \mathrm{~A}-\mathrm{H} 3 \mathrm{~A} 1$ & 110.0 & $\mathrm{O} 2 \mathrm{~B}^{\mathrm{ii}}-\mathrm{C} 11 \mathrm{~B}-\mathrm{O} 2 \mathrm{~B}$ & $159.4(5)$ \\
\hline $\mathrm{Cr} 1-\mathrm{N} 3 \mathrm{~A}-\mathrm{H} 3 \mathrm{~A} 1$ & 110.0 & $\mathrm{O} 2 \mathrm{C}-\mathrm{ii} 11 \mathrm{C}-\mathrm{O} 2 \mathrm{C}$ & $92.7(9)$ \\
\hline $\mathrm{C} 3 \mathrm{~A}-\mathrm{N} 3 \mathrm{~A}-\mathrm{H} 3 \mathrm{~A} 2$ & 110.0 & $\mathrm{O} 2 \mathrm{C}^{\mathrm{ii}}-\mathrm{C} 11 \mathrm{C}-\mathrm{O} 1 \mathrm{C}^{\mathrm{ii}}$ & $86.8(6)$ \\
\hline $\mathrm{Cr} 1-\mathrm{N} 3 \mathrm{~A}-\mathrm{H} 3 \mathrm{~A} 2$ & 110.0 & $\mathrm{O} 2 \mathrm{C}-\mathrm{C} 11 \mathrm{C}-\mathrm{O} 1 \mathrm{C}^{\mathrm{ii}}$ & $79.0(6)$ \\
\hline $\mathrm{H} 3 \mathrm{~A} 1-\mathrm{N} 3 \mathrm{~A}-\mathrm{H} 3 \mathrm{~A} 2$ & 108.4 & $\mathrm{O} 2 \mathrm{C}-\mathrm{ii}-\mathrm{C} 1 \mathrm{C}-\mathrm{O} 1 \mathrm{C}$ & $79.0(6)$ \\
\hline $\mathrm{N} 2 \mathrm{~A}-\mathrm{C} 2 \mathrm{~A}-\mathrm{C} 3 \mathrm{~A}$ & $106.7(9)$ & $\mathrm{O} 2 \mathrm{C}-\mathrm{C} 11 \mathrm{C}-\mathrm{O} 1 \mathrm{C}$ & $86.8(6)$ \\
\hline $\mathrm{N} 2 \mathrm{~A}-\mathrm{C} 2 \mathrm{~A}-\mathrm{H} 2 \mathrm{~A} 3$ & 110.4 & $\mathrm{O} 1 \mathrm{C} i-\mathrm{C} 11 \mathrm{C}-\mathrm{O} 1 \mathrm{C}$ & $159.4(5)$ \\
\hline $\mathrm{C} 3 \mathrm{~A}-\mathrm{C} 2 \mathrm{~A}-\mathrm{H} 2 \mathrm{~A} 3$ & 110.4 & & \\
\hline $\mathrm{Cr} 1-\mathrm{N} 2 \mathrm{~A}-\mathrm{C} 2 \mathrm{~A}-\mathrm{C} 3 \mathrm{~A}$ & $-42.0(11)$ & $\mathrm{Cr} 1-\mathrm{N} 2 \mathrm{~B}-\mathrm{C} 2 \mathrm{~B}-\mathrm{C} 3 \mathrm{~B}$ & $44.2(13)$ \\
\hline
\end{tabular}




\section{supporting information}

$\begin{array}{llll}\mathrm{Cr} 1-\mathrm{N} 3 \mathrm{~A}-\mathrm{C} 3 \mathrm{~A}-\mathrm{C} 2 \mathrm{~A} & -44.8(13) & \mathrm{N} 2 \mathrm{~B}-\mathrm{C} 2 \mathrm{~B}-\mathrm{C} 3 \mathrm{~B}-\mathrm{N} 3 \mathrm{~B} & -56.3(15) \\ \mathrm{N} 2 \mathrm{~A}-\mathrm{C} 2 \mathrm{~A}-\mathrm{C} 3 \mathrm{~A}-\mathrm{N} 3 \mathrm{~A} & 57.9(14) & \mathrm{Cr} 1-\mathrm{N} 3 \mathrm{~B}-\mathrm{C} 3 \mathrm{~B}-\mathrm{C} 2 \mathrm{~B} & 40.2(12)\end{array}$

Symmetry codes: (i) $-x+1 / 2,-y+1 / 2,-z+1$; (ii) $-x+1, y,-z+3 / 2$.

Hydrogen-bond geometry $\left(A,{ }^{\circ}\right)$

\begin{tabular}{lllll}
\hline$D-\mathrm{H} \cdots A$ & $D-\mathrm{H}$ & $\mathrm{H} \cdots A$ & $D \cdots A$ & $D-\mathrm{H} \cdots A$ \\
\hline $\mathrm{N} 2 A-\mathrm{H} 2 A 1 \cdots \mathrm{S} 1^{\mathrm{iii}}$ & 0.89 & 2.45 & $3.324(17)$ & 167 \\
$\mathrm{~N} 2 A-\mathrm{H} 2 A 2 \cdots \mathrm{O} 2 B^{\mathrm{iv}}$ & 0.89 & 2.41 & $3.187(19)$ & 146 \\
$\mathrm{~N} 3 A-\mathrm{H} 3 A 1 \cdots \mathrm{O} 1 B^{\mathrm{v}}$ & 0.89 & 2.58 & $3.282(16)$ & 136 \\
$\mathrm{~N} 2 B-\mathrm{H} 2 B 1 \cdots \mathrm{S} 1^{\mathrm{iii}}$ & 0.89 & 2.77 & $3.459(17)$ & 135 \\
$\mathrm{~N} 3 B-\mathrm{H} 3 B \cdots \mathrm{O} 2 C^{\mathrm{v}}$ & 0.89 & 2.45 & $3.22(2)$ & 145 \\
$\mathrm{~N} 3 B-\mathrm{H} 3 B 2 \cdots \mathrm{S} 1^{\mathrm{vi}}$ & 0.89 & 2.38 & $3.255(18)$ & 166
\end{tabular}

Symmetry codes: (iii) $-x+1 / 2, y-1 / 2,-z+3 / 2$; (iv) $x, y-1, z$; (v) $x,-y+1, z-1 / 2$; (vi) $-x+1 / 2,-y+3 / 2,-z+1$. 